\title{
Microscopic ion fluxes in plasma-aided nanofabrication of ordered carbon nanotip structures
}

\author{
I. Levchenko ${ }^{\text {a) }}$ and K. Ostrikov ${ }^{\text {b) }}$ \\ School of Physics, The University of Sydney, Sydney, New South Wales 2006, Australia \\ M. Keidar ${ }^{\text {c) }}$ \\ Department of Aerospace Engineering, University of Michigan, Ann Arbor, Michigan 48109-2140 \\ S. $X u^{\text {d) }}$ \\ Plasma Sources and Applications Center, National Institute of Education (NIE), Nanyang Technological \\ University, 637616 Singapore
}

(Received 25 February 2005; accepted 29 July 2005; published online 23 September 2005)

\begin{abstract}
Three-dimensional topography of microscopic ion fluxes in the reactive hydrocarbon-based plasma-aided nanofabrication of ordered arrays of vertically aligned single-crystalline carbon nanotip microemitter structures is simulated by using a Monte Carlo technique. The individual ion trajectories are computed by integrating the ion equations of motion in the electrostatic field created by a biased nanostructured substrate. It is shown that the ion flux focusing onto carbon nanotips is more efficient under the conditions of low potential drop $U_{s}$ across the near-substrate plasma sheath. Under low- $U_{s}$ conditions, the ion current density onto the surface of individual nanotips is higher for higher-aspect-ratio nanotips and can exceed the mean ion current density onto the entire nanopattern in up to approximately five times. This effect becomes less pronounced with increasing the substrate bias, with the mean relative enhancement of the ion current density $\xi_{i}$ not exceeding $\sim 1.7$. The value of $\xi_{i}$ is higher in denser plasmas and behaves differently with the electron temperature $T_{e}$ depending on the substrate bias. When the substrate bias is low, $\xi_{i}$ decreases with $T_{e}$, with the opposite tendency under higher- $U_{s}$ conditions. The results are relevant to the plasma-enhanced chemical-vapor deposition of ordered large-area nanopatterns of vertically aligned carbon nanotips, nanofibers, and nanopyramidal microemitter structures for flat-panel display applications. (C) 2005 American Institute of Physics. [DOI: 10.1063/1.2040000]
\end{abstract}

\section{INTRODUCTION}

Surface-conduction electron-emitter display (SED) technology is one of the most recent advances in large flat-panel display manufacturing, with the expected market forecast exceeding US $\$ 30$ billion in 2007. ${ }^{1}$ A field-emission display (FED) technology, one of the derivatives of the SED, is based on ordered arrays of microemitters, each producing an individual pixel. The combined multipixel emission from the microemitter array is expected to generate exotic color patterns with millions of individual colors and superior color reproduction and darkness contrast. The FED technology is one of the most promising advances on the way to manufacture ultrathin large-area flat-panel displays. The emitter material usually contains ordered patterns of electron emitters on its surface. Various quasi-one-dimensional carbon-based nanostructures, such as carbon nanofibers, nanopyramidal structures, multiwalled carbon nanotubes, and several others, are promising for microemitter applications owing to their outstanding size-dependent electronic properties, geometric

\footnotetext{
a) Permanent address: National Aerospace University, Kharkov, Ukraine.; electronic mail: iglev@ukr.net

${ }^{\text {b) }}$ Author to whom correspondence should be addressed; Also at: Plasma Sources and Applications Center, NIE, Nanyang Technological University, 637616 Singapore; electronic mail: K.Ostrikov@physics.usyd.edu.au

c)Electronic mail: keidar@engin.umich.edu

${ }^{\mathrm{d})}$ Electronic mail: syxu@nie.edu.sg
}

field-enhancing factors such as high geometric aspect ratio, and excellent conductivity, ${ }^{2-7}$ and have recently been commonly recognized as an important alternative to conventional electronic materials. ${ }^{8-13}$

Plasma-enhanced chemical-vapor deposition (PECVD) is one the most efficient and precise tools of fabrication of the ordered arrays of carbon-based microemitters. ${ }^{14-23}$ Most recently, the possibility of plasma-aided nanofabrication of large-area ordered patterns of single-crystalline carbon nanotips (high-aspect-ratio nanosized conical structures) with excellent field-emitting properties (high emission currents at low turn-on voltages) has been reported. ${ }^{24}$ However, relatively low emission currents, poor coherence in the emission pattern, and difficulties in integration into very-large-scaleintegrated silicon-based microdevices ${ }^{25}$ impede the overall progress in the carbon nanostructure- (CNS) based nanodevice fabrication and remain a major challenge for the $R \& D$ efforts in the coming years. Relevant problems can arizes, e.g., due to imperfections in the structure, sizes, orientations, alignment, and ordering of the CNS patterns, and become critical for upscaling the nanostructured patterns to larger surface areas. It is thus imperative to be able to control the plasma-aided nanofarication process to ensure that the device quality of the individual nanostructures and their ordered arrays is met. ${ }^{26}$

A possible solution can be devised by noting that the process proceeds via the "top-down" nanoassembly ${ }^{27-29}$ 
powered by the reactive plasma ions driven towards the nanostructured deposition surface by a dc electric field sustained in the near-substrate plasma sheath. Specifically, recent results of the $a b$ initio local-density approximationbased density-functional theory computations of the equilibrium chemical structure of the single-crystalline carbon nanotip microemitters reveal a crucial role of $\mathrm{CH}_{3}^{+}$cationic building units (BUs) that build up an equilibrium carbon network peripherally terminated by hydrogen atoms. ${ }^{30}$ To this end, controllable and uniform over large areas precipitation of cationic BUs is widely believed to be an efficient strategy for success. This requirement is common in most of the plasma-assisted materials synthesis and surface modification technologies. $^{31,32}$

However, nanoscale fabrication imposes one more essential requirement on the ability to control the distribution of cation fluxes in the immediate vicinity (typically at distances $\leqslant 100 \mathrm{~nm}$ ) of the nanotip surfaces required to maintain a high degree of three-dimensional positional uniformity of the growth pattern. Therefore, modeling microscopic deposition fluxes of cationic BUs on the nanotip surfaces is a critical issue in the development of control strategies of relevant nanofabrication processes and is the main aim of this work.

It has previously been reported that the vertically aligned nanotips feature a base radius varying from 10 to $100 \mathrm{~nm}$, and height in the range of $100-400 \mathrm{~nm}^{33-35}$ Furthermore, the field-emission characteristics strongly depend on the CNS geometrical parameters. ${ }^{34}$ Specifically, the rarefied nanotip patterns consisting of larger individual nanostructures grown at higher substrate temperature show the highest emissive current. Noteworthy, the ion deposition from the low-pressure chemically active plasmas of $\mathrm{Ar}+\mathrm{H}_{2}$ $+\mathrm{CH}_{4} / \mathrm{C}_{2} \mathrm{H}_{2}$ gas mixtures is a very efficient technique of the plasma-aided fabrication of carbon nanotip microemitter arrays. ${ }^{15,16,19,20,24,33-35}$ This method allows one to control the key deposition parameters, such as the ion energy and flux composition, as well as the deposition substrate temperature $T_{s}$, with the possibility to control $T_{s}$ by the fluxes of charged and neutral species from the plasma bulk. In this technique, the ions are accelerated in the sheath area separating the plasma bulk and the substrate surface. ${ }^{31,32,36}$ It is notable that under prevailing deposition conditions, ${ }^{33-35}$ the fluxes of $\mathrm{C}_{x} \mathrm{H}_{y}^{+}$cation radicals at the plasma sheath boundary can exceed the fluxes of neutral radicals. ${ }^{33}$ However, despite a remarkable progress in the synthesis and growth optimization of the CNSs in hydrocarbon-based plasmas, the existing numerical efforts are mostly limited to modeling of the plasma species composition and other discharge parameters. ${ }^{33,37-39}$

In this article, the microscopic topology of the ion current distribution in the vicinity of the ordered patterns of carbon nanotip structures is studied. In this case, the ion energy is controlled by the (negative with respect to the plasma bulk) substrate bias, which usually varies from several tens of volts (for self-biased substrates) to $150-200 \mathrm{~V}$ (Ref. 33) [and in some cases of externally unheated substrates to 300-400 V (Ref. 35)] when an external dc substrate bias is used. Thus, the energetic cation fluxes efficiently heat and activate the catalyzed substrate surface,

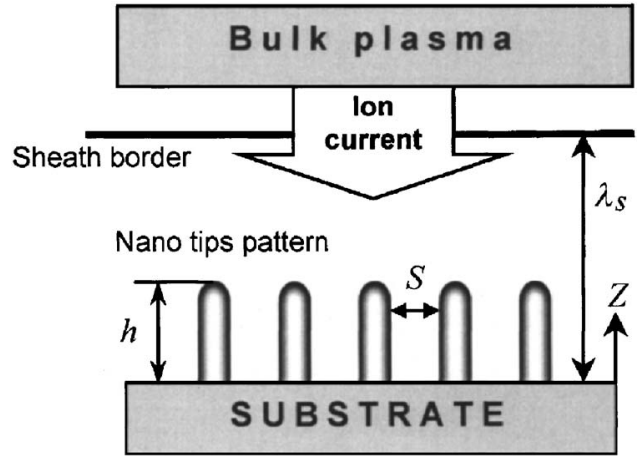

FIG. 1. Schematic representation of the simulation geometry in the process of PECVD of ordered carbon nanotip patterns.

eventually giving rise to the growth of aligned and ordered nanostructures. Here, we present the results of the Monte Carlo (MC) numerical simulation of the ion current distribution in the ordered carbon nanotip structures and demonstrate that the microscopic ion flux distribution over the nanotip surfaces can be efficiently controlled by the dc substrate bias, ion density, and electron temperature.

The paper is organized as follows. In Sec. II, a model for numerical simulation of the ion current-density distribution in ordered carbon nanotip patterns is presented. In Sec. III the numerical simulation technique and initial and boundary conditions are described. The numerical results are presented in Sec. IV. Section V is devoted to the interpretation and implications of the simulation results. The paper concludes with a summary of the results obtained and a brief outlook for future research.

\section{FORMULATION AND MODEL}

Consider a system consisting of a biased substrate and the plasma bulk, wherein the ion flux is extracted and deposited on the substrate surface (Fig. 1). We emphasize that the details of the plasma generation are not essential in the case considered; apart from the low-pressure rf plasmas of $\mathrm{Ar}$ $+\mathrm{H}_{2}+\mathrm{CH}_{4} / \mathrm{C}_{2} \mathrm{H}_{2}$ gas mixtures mentioned above, the plasma can be produced by any conventional plasma sources such as cathodic vacuum arcs, ${ }^{36,40} \mathrm{dc},{ }^{41}$ capacitive rf discharges, ${ }^{42}$ and microwave ${ }^{43}$ discharges. For simplicity, only a single cationic species $\mathrm{CH}_{3}^{+}$(assumed the main $\mathrm{BU}$ of the nanostructures in question) is considered in computations. A thin sheath separates the substrate and the plasma bulk as shown in Fig. 1. As the potential drop in the presheath area is rather small (e.g., in the plasma facility ${ }^{44}$ used for the PECVD of CNSs (Refs. 33-35) it is $\sim T_{e} / 2 \sim 1-2.5 \mathrm{eV}$, where $T_{e}$ is the electron temperature), only the actual potential difference across the plasma sheath is taken into consideration here. Furthermore, the ions are assumed to enter the sheath at $z$ $=\lambda_{s}$ with the Bohm velocity $V_{B}=\left(T_{e} / m_{i}\right)^{1 / 2}$, where $m_{i}$ is the ion mass, and $\lambda_{s}$ is the sheath thickness, and accelerated in the sheath to the energy equal to the cross-sheath potential drop $U_{s}$.

When the cross-sheath potential drop is relatively high $\left(T_{e} \ll U_{s}\right)$, one has an estimate for the sheath thickness ${ }^{32}$ 


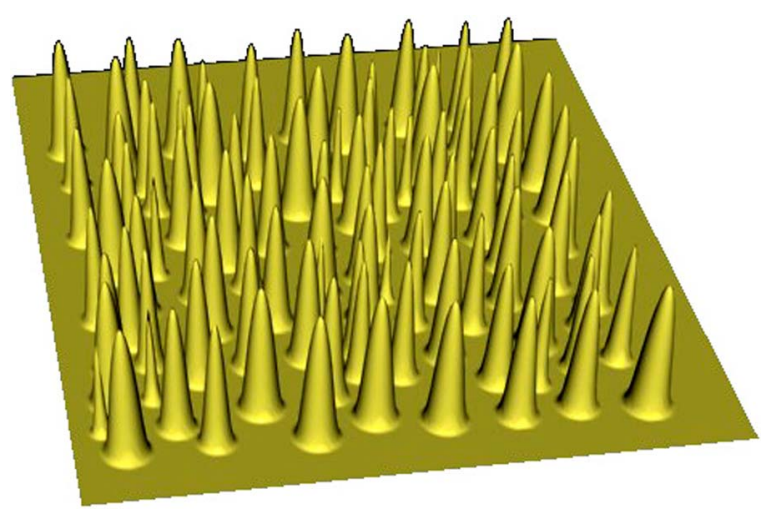

FIG. 2. (Color online) Ordered pattern of carbon nanotip structures used in numerical simulations.

$$
\lambda_{s}=\frac{\sqrt{2}}{3} \lambda_{D}\left(\frac{2 U_{s}}{T_{e}}\right)^{3 / 4}
$$

where $\lambda_{D}$ is the electron Debye length. On the other hand, when the external bias is low or the substrate is floating $\left(T_{e} \sim U_{s}\right)$, it is quite accurate to assume that the sheath is of the order of a few Debye lengths

$$
\lambda_{s}=\gamma_{s} \lambda_{D}=\gamma_{s}\left(\frac{\varepsilon_{0} T_{e}}{e n_{p}}\right)^{1 / 2}
$$

where $\varepsilon_{0}$ is a dielectric constant, $n_{p}$ is the plasma density (which in the single-ion-species approximation is either the electron $n_{e}$ or ion $n_{i}$ number density), and $\gamma_{s}$ is a constant, typically in the range between 1 and $5 .^{32}$

The microscopic topography of the ion current has been simulated for the nanotip pattern shown in Fig. 2. It has been assumed that the deposition surface is covered with an ordered array of nanotips with the radius $R$ varying from 20 to $70 \mathrm{~nm}$ (mean radius $R_{m}$ is equal to $44 \mathrm{~nm}$ ), height $h$ $=300 \mathrm{~nm}$, and mean spacing (distance between adjacent nanostructures) $S_{m}=100 \mathrm{~nm}$ representative to PECVD of carbon nanotip structures. ${ }^{24,33-35}$ The distribution of the nanotip radii in the simulation is shown in Fig. 3. We have further assumed that the entire simulated surface, including surfaces of CNSs, is conductive, which is relevant to the PECVD of

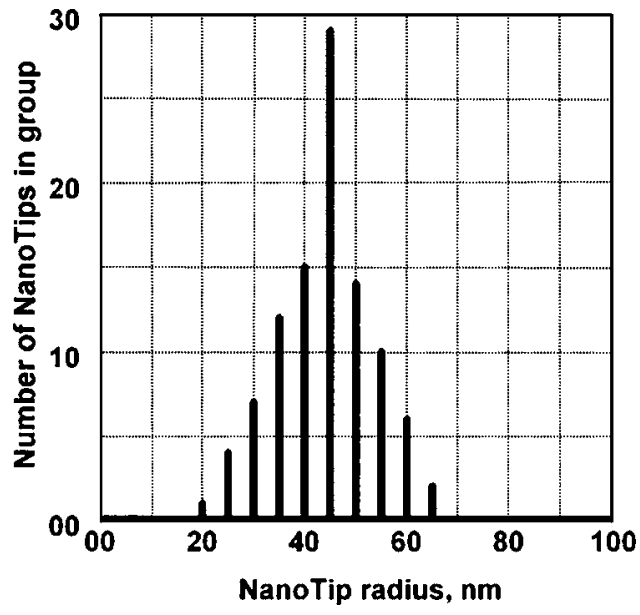

FIG. 3. Distribution of radii of carbon nanotips in the simulation pattern of Fig. 2. carbon nanotips on nickel-catalyzed highly doped $\mathrm{Si}(100)$ substrates in the experiments of our interest here.

In this case, the electric field is strongly nonuniform in the vicinity of the CNS tips, and the ion motion is determined by the effective electric field $\mathbf{E}$ of the entire nanotip pattern of Fig. 2. The ion motion in the field $\mathbf{E}$ can be described by the following equations of motion:

$$
\mathbf{r}(t)=\mathbf{r}_{0}+\int_{t_{0}}^{t}\left[\mathbf{v}_{0}+\int_{\tau_{0}}^{0} \frac{\mathbf{E}(r, \tau)}{m_{i}} d r\right] d \tau,
$$

where $\mathbf{r}(t)$ is the ion position vector, $\mathbf{r}_{0}$ is the initial position vector for an ion located at the sheath border $z=\lambda_{s}, t_{0}$ is the initial time moment of ion motion, and $\mathbf{v}_{0}=V_{B}$ is the ion velocity at the sheath edge. Equation (3) can be used for explicit integration of the ion trajectories, provided that the electric-field distribution in the proximity of the nanopatterned substrate is known. The surface charges on the nanotips have been calculated by approximating the nanotip surface as a hollow cylinder capped with a semisphere and using conventional electrodynamics for electrostatic potentials of the above structures. ${ }^{45}$ The effective electric field acting on an ion located at the space point $\mathbf{r}$ has been computed by integrating the surface charge of all nanotips in the pattern:

$$
\mathbf{E}(\mathbf{r})=\sum_{i=1}^{n} \int_{S_{i}} \frac{\sigma_{i} d S_{i} \mathbf{r}}{4 \pi \varepsilon_{0} r^{3}}+\mathbf{E}_{S},
$$

where $\sigma_{i}$ is the surface density of electric charge on the nanotip surfaces, $S_{i}$ is the surface area of the $i$ th nanotip, and $\mathbf{E}_{S}=-\nabla \phi$ is the electric field in the plasma sheath area, corresponding to the quasi-one-dimensional parabolic potential profile $^{32}$

$$
\phi(z)=U_{s}\left(z / \lambda_{s}\right)^{4 / 3},
$$

between the biased substrate and the plasma bulk. Since $h$ $\ll \lambda_{s}$, the ion motion in the plasma sheath is described by Eq. (5), whereas Eq. (4) is used to simulate the ion motion in the vicinity of the nanopattern in Fig. 2.

\section{BOUNDARY CONDITIONS AND NUMERICAL METHOD}

The ion motion towards the nanopatterned substrate has been simulated in the simulation space bounded by the curved surface of the nanopattern in Fig. 2 and the plane $z=\lambda_{s}$ in Fig. 1. In the $x$ and $y$ directions (the substrate surface plane), the simulation space was bounded by the rectangular substrate area of $2.5 \times 2.5 \mu \mathrm{m}^{-3}$ comprising 100 individual nanotips. The simulation set of the plasma and nanostructure parameters, representative to the low-frequency $(\sim 460 \mathrm{kHz})$ inductively coupled plasma source ${ }^{44}$ and the PECVD of CNSs,${ }^{33-35}$ is summarized in Table I.

The ion motion has been simulated by using the following routine. First, the sheath thickness $\lambda_{s}$ was calculated by using Eqs. (1) or (2), depending on the electron thermal energy and cross-sheath potential assumed. Meanwhile, the surface charge for each nanotip in the ensemble, as well as the potential distribution in the sheath were computed from Eqs. 
TABLE I. Parameters and representative values in computations.

\begin{tabular}{|c|c|c|}
\hline Parameter & Notation & Value \\
\hline Carbon nanotip radius & $R$ & $20-70 \mathrm{~nm}$ \\
\hline Mean radius of nanotips & $R_{m}$ & $44 \mathrm{~nm}$ \\
\hline Nanotip height & $h$ & $300 \mathrm{~nm}$ \\
\hline Spacing between nanotips & $S$ & $50-150 \mathrm{~nm}$ \\
\hline Mean spacing between nanotips & $S_{m}$ & $100 \mathrm{~nm}$ \\
\hline Number of nanotips & $N_{\mathrm{CNT}}$ & 100 \\
\hline Substrate dimensions & $l_{s} \times l_{s}$ & $2.5 \times 2.5 \mu \mathrm{m}$ \\
\hline Cross-sheath potential drop & $U_{s}$ & $-20,-50 \mathrm{~V}$ \\
\hline Mass of ionic building units & $m_{i}$ & $15 \mathrm{amu}$ \\
\hline Electron temperature & $T_{e}$ & $1.0-12.0 \mathrm{eV}$ \\
\hline Plasma density & $n_{p}$ & $10^{17}-5 \times 10^{18} \mathrm{~m}^{-3}$ \\
\hline Number of ions in simulation & $N_{i}$ & $3 \times 10^{5}$ \\
\hline Relative enhancement of ion current density & $\xi_{i}$ & $1-5$ \\
\hline Debye length & $\lambda_{D}$ & $8 \times 10^{-4}-2 \times 10^{-2} \mathrm{~cm}$ \\
\hline Sheath constant & $\gamma_{s}$ & $1-5$ \\
\hline Ion energy at the sheath edge & $\varepsilon_{i}$ & $1.0-12.0 \mathrm{eV}$ \\
\hline $\begin{array}{l}\text { Distance between equidistant planes } \\
\text { used for quantifying ion current distribution } \\
\text { along lateral surfaces of nanotips }\end{array}$ & $d_{\text {lat }}^{-}$ & $6 \mathrm{~nm}$ \\
\hline Magnitude of electric field & $E$ & $10^{7} \mathrm{~V} / \mathrm{m}$ \\
\hline Ion velocity at the sheath edge & $V_{B}$ & $2.5-4 \times 10^{3} \mathrm{~m} / \mathrm{s}$ \\
\hline
\end{tabular}

(4) and (5). The obtained values were further used in the Monte Carlo simulation of the ion current distribution. Second, the ion flux distribution was computed by the MC simulation of each separate ion trajectory. An initial position of all the ions was set randomly in the $z=\lambda_{s}$ parallel to the substrate (i.e., at the interface between the plasma bulk and the sheath area). Thereafter, the ion motion was simulated over a number of temporal steps and stopped when the ion collided with any of the surfaces (substrate or nanotip) in the nanopattern of Fig. 2. Since the ions were traced separately one after another, a mutual influence of the ion charges was accounted indirectly, by using the relation for potential distribution (5) valid for the nonzero space-charge conditions. Thus, the results obtained are applicable to large enough ion current densities that cannot be neglected. ${ }^{46}$ The coordinates $x, y$, and $z$ of the ion collisions with the nanopattern were input, together with the unique ion number $i$, into a database. For the next simulation step, an ion was introduced randomly on the sheath boundary. The total number of ions $N_{i}$ used in the simulations was $3 \times 10^{5}$ for each set of the parameters (plasma density, substrate bias, etc.). We recall that the ion energy at the sheath edge was assumed $\mathcal{E}_{i} \approx m_{i} V_{B}^{2} / 2$.

Thus, as a result of the simulation, the database with the coordinates of the ion collisions with the substrate and nanotip surfaces was created. Subsequent data processing allowed us to compute the ion current distributions over lateral surfaces of individual nanotips of different radii and other important characteristics of the ion-nanotip interaction such as the mean height of deposition and mean ion displacement.

\section{NUMERICAL RESULTS}

In this section, we consider the effect of the plasma sheath width and other process parameters on the ion flux distribution over the nanostructured pattern of Fig. 2. The narrow sheath case considered in Sec. IV A corresponds to the conditions of Eq. (2), whereas the wide sheath case of Sec. IV B to Eq. (1).

\section{A. Narrow sheath}

The narrow sheath case has been simulated by using $U_{s}=20 \mathrm{~V}$ for the cross-sheath potential drop. We recall that such conditions correspond to floating or weakly biased substrates. A typical ion current distribution onto the substrate surface is shown in Fig. 4. From Fig. 4, one can notice that the ion current is strongly focused by the nanotips (ion current spikes correspond to the nanotip positions). Indeed, the electric field is nonuniform in the vicinity of sharp tips and deflects the ions from straight trajectories causing their nonuniform precipitation to the nanotip lateral surfaces in the areas closer to their upper ends. We note that in the simulation, each ion from the ensemble was traced over an indi-

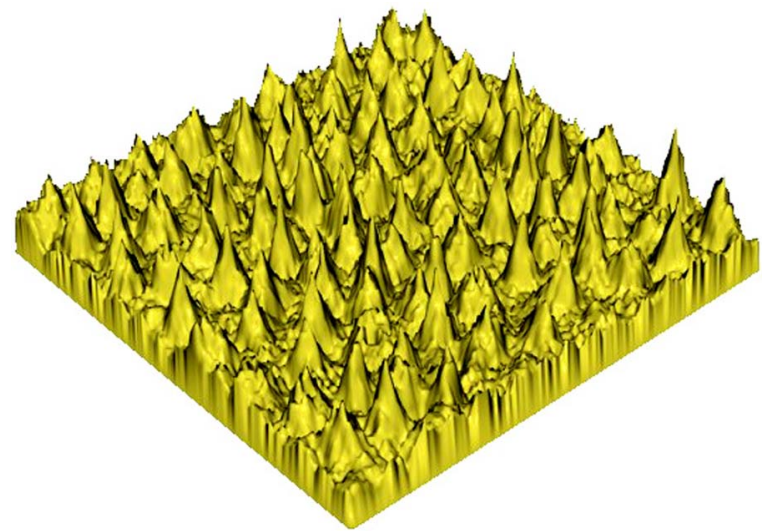

FIG. 4. (Color online) Representative three-dimensional topography of ion current distribution over the nanostructured surface for $U_{s}=20 \mathrm{~V}, n_{p}$ $=5 \times 10^{17} \mathrm{~m}^{-3}$, and $T_{e}=2 \mathrm{eV}$. 

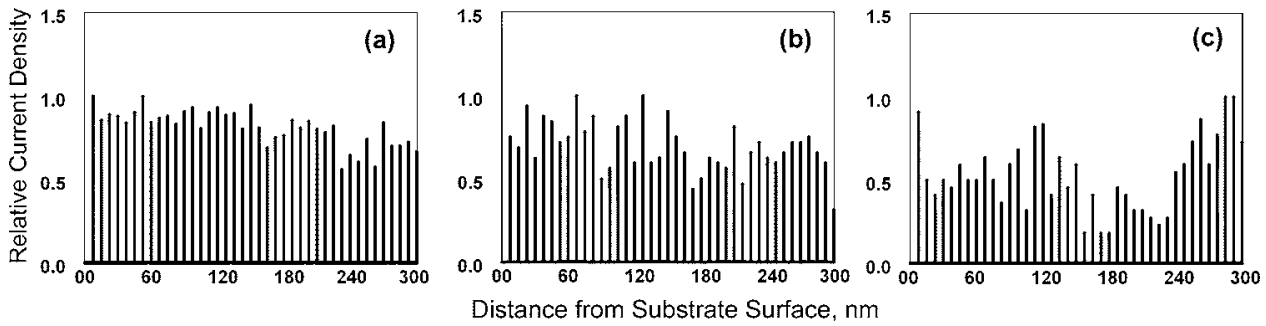

FIG. 5. Distribution of relative ion current density along the nanotip lateral surface as a function of distance from the substrate surface $z$ for $T_{e}=2 \mathrm{eV}$ and $U_{s}=20 \mathrm{~V}$. Diagrams (a)-(c) correspond to the following values of the plasma density $1.7 \times 10^{18}, 8.6 \times 10^{17}$, and $1.23 \times 10^{17} \mathrm{~m}^{-3}$, respectively.

vidual trajectory with a randomly chosen starting point. This can be regarded as one of the reasons of the stochasticlike pattern of the ion current distribution in Fig. 4.

As we have mentioned above, the $z$ coordinates of the points of the ion collisions with the nanotips were also recorded in the process of simulation and subsequently used to study the ion current-density distribution along the nanotip lateral surfaces. To quantify this effect, the surface of each nanotip was split into 50 areas by equidistant parallel planes $z=k d_{\text {lat }}$, where $d_{\text {lat }}=6 \mathrm{~nm}$, and $k=1.49$ is an integer. Accordingly, all the ions deposited on the nanotip lateral surfaces (excluding the ions precipitated to the substrate surface free of nanotips) were divided into 50 groups, according to their specific area of collision with the nanotip.

The histograms in Fig. 5 suggest that the distribution of the ion current density over the nanotip lateral surfaces depends on the plasma density. From Fig. 5(a), one can notice that when the plasma density is higher, the ion current to the nanotip lateral surface is distributed fairly uniformly. In this case the ion flux is somewhat higher in the surface areas close to the nanotip base. In less dense plasmas, the ion flux distribution becomes less uniform as can be seen in Fig. 5(b). Likewise, the position of the maximum of the ion flux still remains closer to the substrate surface. On the other hand, when $n_{p}$ is reduced by one order of magnitude, two poorly resolved peaks located closer to the ends of the nanostructures can be observed [Fig. 5(c)].

Figure 6 displays the dependence of the relative ion current density to the nanotips (i.e., the ratio of the densities of the ion current to the nanotips and the mean ion current to the entire simulation area in the $x-y$ plane) on the nanotip radius $R$, with the plasma density as a parameter. The graphs in Fig. 6 have been plotted by using the following routine. First, the relative ion current density was calculated for each individual nanotip by dividing the density of the total current to the nanotip by the density of the total current to the substrate. Then, all points were plotted as a function of the nanotip radius. Thus, multiple points in Fig. 6 for the same nanotip radius reflect the position-dependent dispersion of the ion current density to the nanotips of the same radius. One can see that the ion current focusing is much stronger in the higher-density plasma [Fig. 6(a)]. Furthermore, the nanotips of a smaller radius focus the ion current more efficiently. For instance, the relative ion current density onto the nanotips with $R \sim 20 \mathrm{~nm}$ in six to eight times exceeds the mean current density. However, the ion current density onto larger nanotips $(R \sim 60 \mathrm{~nm})$ is almost twice the mean value. In the lower-density plasma, the current focusing is less pro- nounced and reaches 3 and 1.5 for the nanotips with $R \sim 20$ and $60 \mathrm{~nm}$, respectively. Besides, one can note that the relative dispersion of the ion current density is small in dense plasmas (up to $15 \%$ of the ion current density), increases when $n_{p}$ is reduced, and reaches $50 \%$ for $R=30 \%$ and $80 \%$ for $R=40 \mathrm{~nm}$. It is notable that in both high- and low-density cases, the relative dispersion decreases with the nanotip radius approaching its maximum value at $R \sim 65 \mathrm{~nm}$.

The dependence of the mean relative current density to the nanotips on the plasma density and electron temperature is shown in Fig. 7. When the plasma density increases, the relative ion current density increases steadily and reaches 1.3 and 2.3 in the low- and high-density cases, respectively [Fig. 7(a)]. The curve plotted for lower $T_{e}$ shows a higher ion current density. As can be seen from Fig. 7(b), the relative
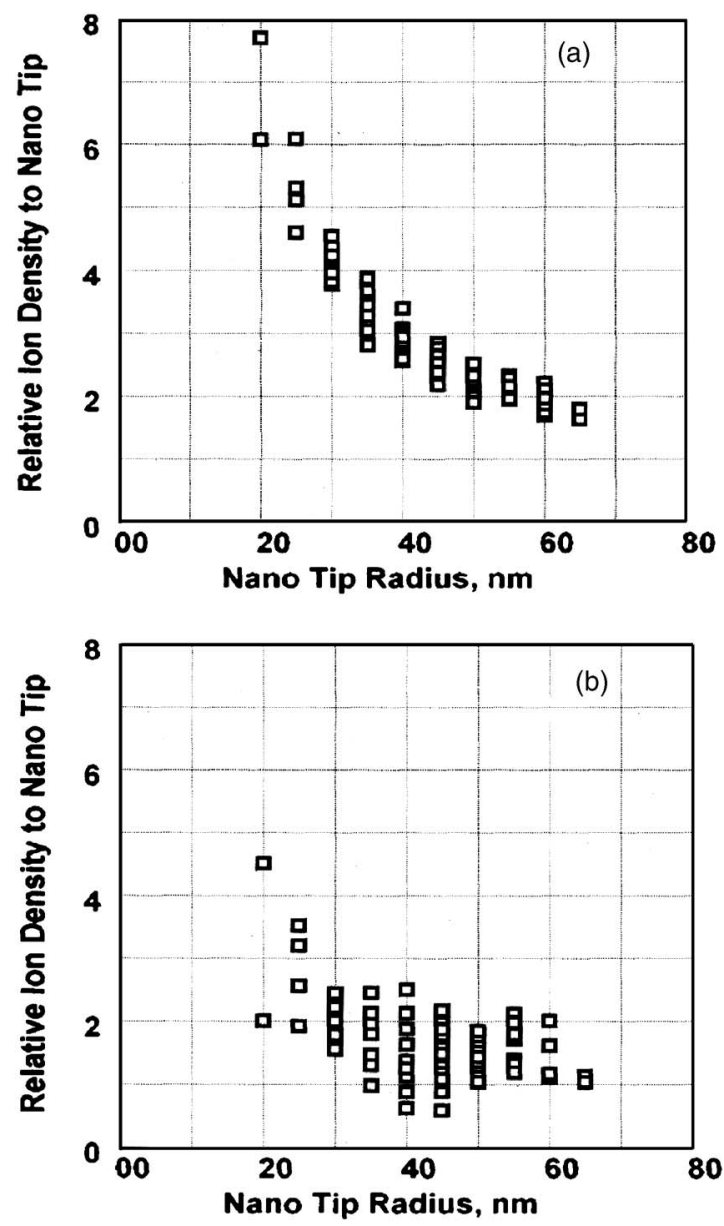

FIG. 6. Relative ion current density on CNTs vs nanotip radius for $U_{s}$ $=20 \mathrm{~V}$ and $n_{p}=1.7 \times 10^{18} \mathrm{~m}^{-3}$ (a) and $1.23 \times 10^{17} \mathrm{~m}^{-3}(\mathrm{~b})$. 

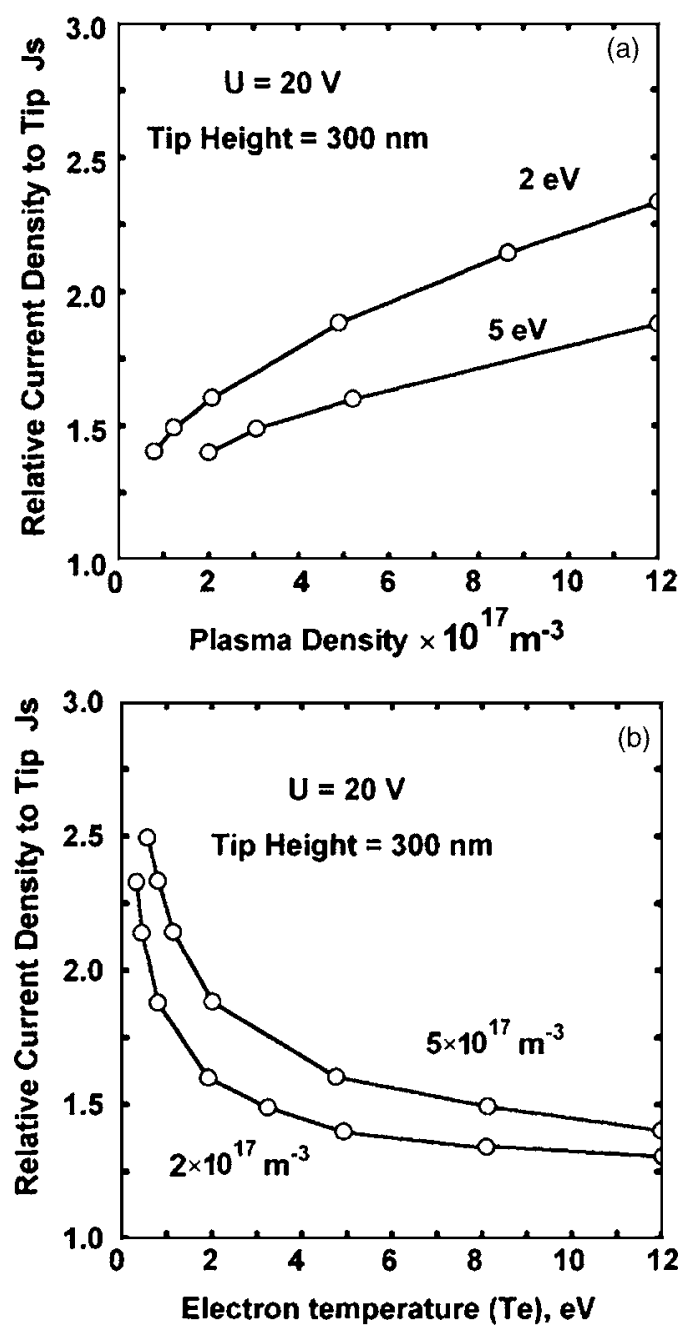

FIG. 7. Relative ion current density to the nanotips as a function of the plasma density (a) and electron temperature (b) for $U_{s}=20 \mathrm{~V}$.

current density decreases steeply in the range of $0.5-5 \mathrm{eV}$, and then decreases smoothly in the subsequent electron temperature range $\left(5 \mathrm{eV}<T_{e}<12 \mathrm{eV}\right)$ leveling off at $T_{e}$ $>12 \mathrm{eV}$. One can also notice from Fig. 7(b) that higher current densities can be obtained at elevated plasma densities.

\section{B. Wide sheath}

To simulate the ion current fluxes in the wide sheath case, we have used a larger potential difference across the sheath $U_{s}=50 \mathrm{~V}$. This case usually requires external biasing of the deposition substrate. Figure 8 shows a representative ion current distribution on the nanostructured surface. A striking observation is that contrary to the narrow sheath case, the ion current focusing is noticeably less efficient. From Fig. 8, one can further notice that the ion current spikes at the nanotip positions become smaller and less resolved compared to the results of Fig. 4. The three-dimensional topography of the ion current is quite stochastic and features numerous fluctuations in the internanotip gap areas.

The histograms in Fig. 9 show that the distribution of the ion current density along the nanotip lateral surface strongly depends on the plasma density. It is seen that when the plasma density is higher [Fig. 9(a)], the maximum of the

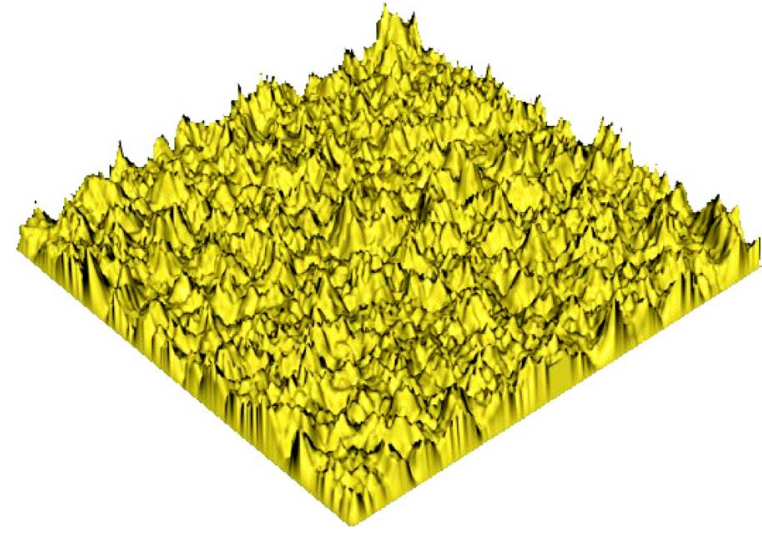

FIG. 8. (Color online) Same as in Fig. 4 for $U_{s}=50 \mathrm{~V}, n_{p}=5 \times 10^{17} \mathrm{~m}^{-3}$, and $T_{e}=2 \mathrm{eV}$.

microscopic ion current is located approximately $180 \mathrm{~nm}$ above the substrate surface, being closer to the top of the nanostructure. However, the current density is much smaller in the upper part of the nanotip structures and diminishes near the tip. On the other hand, $J_{s}$ is almost a quarter of the maximum value near the nanotip base.

In lower-density plasmas, the maximum of the ion density shifts towards the substrate surface, still remaining well shaped. In this case the ion current density to the nanotip base increases, as suggested by Fig. 9(b). Indeed, if $n_{p}$ $=3.8 \times 10^{17} \mathrm{~m}^{-3}$, the maximum of the ion current density is located in the immediate proximity to the substrate surface [Fig. 9(c)]. Furthermore, the ion flux to more distant from the substrate areas becomes almost negligible.

Figure 10 shows the relative ion current density to the nanotips as a function of the tip radius at different values of the plasma density. It is seen that the ion current focusing by the nanotips is generally similar to but less efficient than in the low- $U_{s}$ case shown in Fig. 6. From Fig. 10(a), one can infer that the ion flux focusing is more efficient in denser plasmas and for smaller nanotip structures. Specifically, the relative ion current density to the nanotips with $R \sim 20 \mathrm{~nm}$ is $\sim 2-2.1$ times larger than the mean relative ion current density $J_{s}^{m}=1$. On the other hand, the ion current densities onto larger nanotips from the ensemble approach $J_{s}^{m}$. When the plasma is less dense, the effective current enhancement reaches 1.5 for smaller tips, whereas $J_{s} \sim J_{s}^{m}$ for the larger ones. The dispersion of the ion current density is large enough for both dense and rarefied plasmas and reaches up to $35 \%$ and $50 \%$, respectively. Both cases also feature a consistent decrease of the relative dispersion with the nanotip radius.

The relative current density to the nanotips versus the plasma density and electron temperature is shown in Fig. 11. The dependence of $J_{s}$ on the plasma density is similar to that shown in Fig. 7. In particular, the relative current density increases from 1 for at lower plasma density to 1.2 for higher $n_{p}$ [Fig. 11(a)]. The curve corresponding to the higher electron temperature shows a higher current density, which is opposite to the narrow sheath case of Sec. IV A. This is consistent with the results in Fig. 11(b) showing a remarkable increase of $J_{s}$ with increasing $T_{e}$, which is a different 

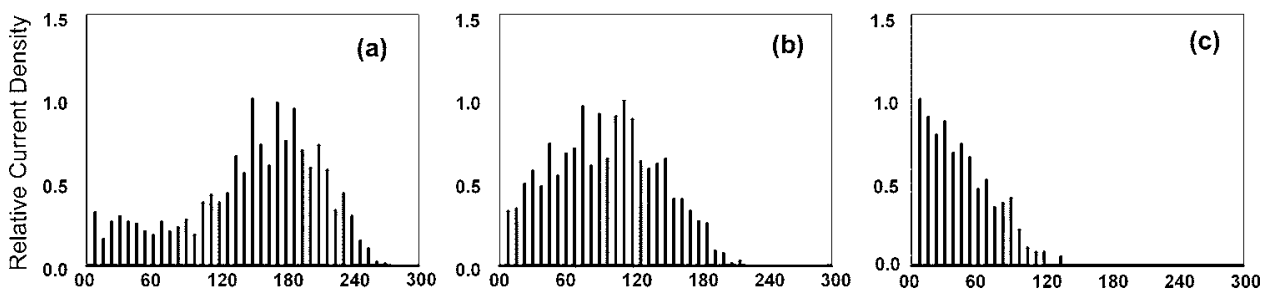

Distance from Substrate Surface, $\mathrm{nm}$

FIG. 9. Same as in Fig. 5 for $T_{e}=2 \mathrm{eV}, U_{s}=50 \mathrm{~V}$, and $n_{p}=2.4 \times 10^{18} \mathrm{~m}^{-3}$ (a), $1.0 \times 10^{18} \mathrm{~m}^{-3}$ (b), and $3.8 \times 10^{17} \mathrm{~m}^{-3}$ (c).

tendency as compared with Fig. 7(b). The rates of increase of the ion current density appear to be different at lower and higher electron temperatures, as can clearly be seen in Fig. 11(b). Meanwhile, the curve corresponding to the higher plasma density shows higher $J_{s}$.

\section{DISCUSSION}

We now attempt to interpret the results reported in Sec. IV and discuss their implications for the development of control strategies of the PECVD of ordered arrays of carbon nanotip microemitters. We will also pinpoint several important considerations for further improvement of the model.

One of the key assumptions of the model adopted here is that the nanotip pattern is conducting and there is no electric charge accumulated on the surface. This assumption is applicable to the case of conducting crystalline nanostructures
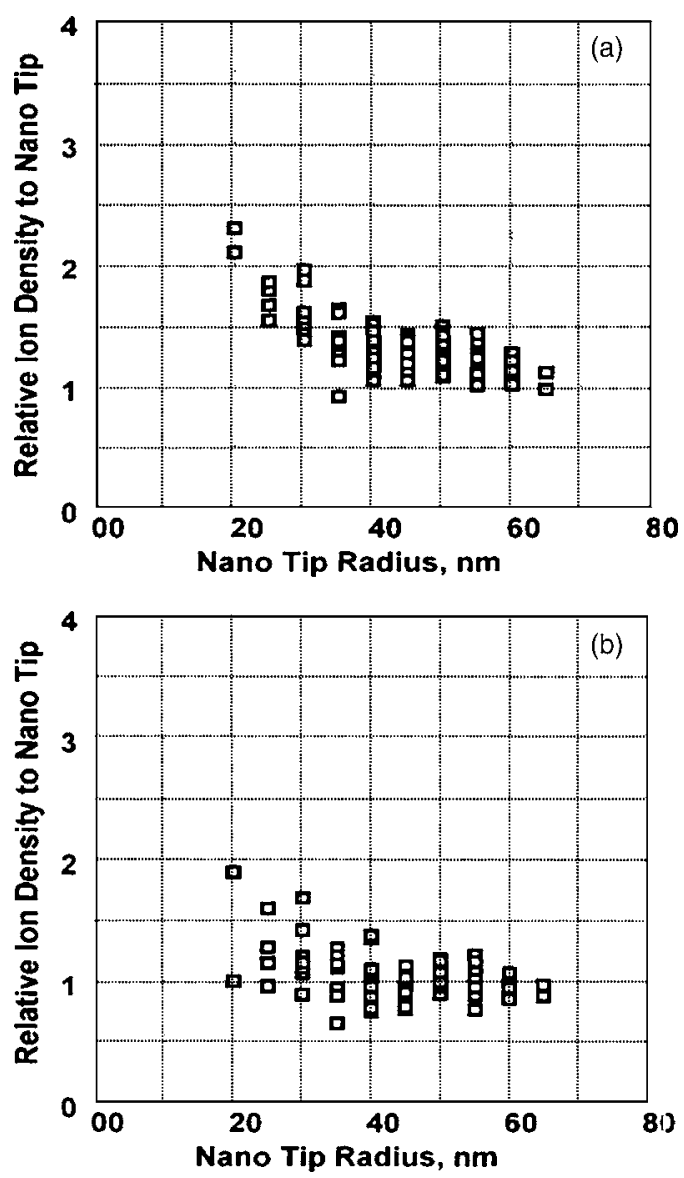

FIG. 10. Same as in Fig. 6 for $U_{s}=50 \mathrm{~V}$ and $n_{p}=2.4 \times 10^{18} \mathrm{~m}^{-3}$ (a) and $2.0 \times 10^{17} \mathrm{~m}^{-3}(\mathrm{~b})$. synthesized through the " base growth" nanoassembly on $\mathrm{Ni} / \mathrm{Fe} / \mathrm{Co}$-catalyzed highly doped silicon substrates. It is worth emphasizing that in other situations the presence of electric charges on the surface can significantly affect the microscopic ion flux distribution over the nanostructured surfaces. For example, ceramic growth islands on the surface can acquire a large positive charge, which can in turn cause quite irregular three-dimensional topographies of the ion flux onto the growth surface. ${ }^{46}$ Interestingly, the distribution of the ion current density onto carbon nanotip structures also shows some signs of irregularity, which is, however, much less pronounced than in the process of growth of dielectric films. Physically, this is attributed to an excellent conductivity of the single-crystalline nanotip structures and a substan-
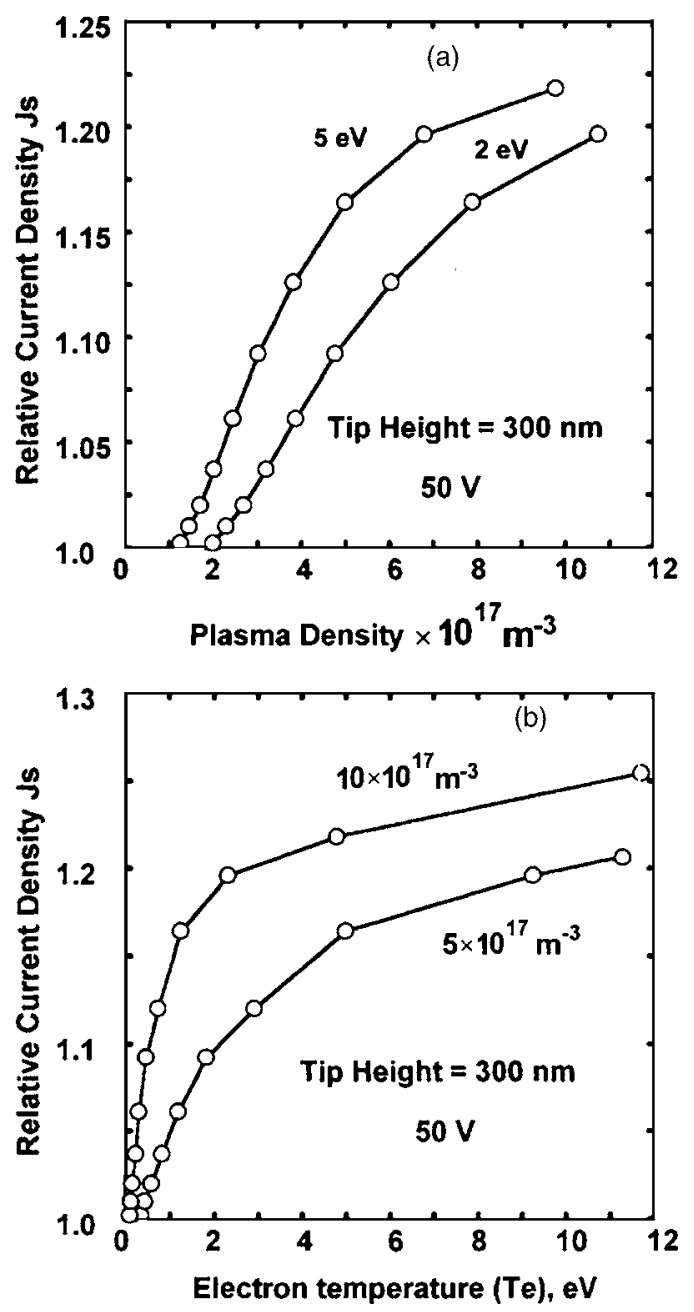

FIG. 11. Same as in Fig. 7 for $U_{s}=50 \mathrm{~V}$. 
tial electric-field enhancement in the proximity of the sharp ends of the CNSs, which strongly affect the ion motion and precipitation.

Another important assumption has been a neglect of ion collisions with other plasma species in the plasma sheath. In this case, nanosized carbon nanotip structures act as the only targets for ion collisions accounted for in the model. This assumption is justified when the rates of ion-neutral, ionelectron, and ion-ion collisions in the plasma sheath are much less than the rate of ion collisions with the surfaces of the nanopattern of Fig. 2. Therefore, the technique adopted here combines the features of the multiple noninteracting single-particle simulation within the plasma sheath and simulation of ion-nanotip collisions at smaller distances, and thus retains a notion of collisional processes commonly attributed to Monte Carlo simulation techniques. ${ }^{47}$

Our results suggest that the irregular electrical field created by the ordered nanotip pattern in most cases affects the distribution of the ion flux onto the substrate and nanotip surfaces. Apparently, the presence of the nanotips on the substrate surface results in a remarkable redistribution of the ion current giving rise to nonuniform and selective ion precipitation. The resulting three-dimensional nonuniform ion deposition patterns (representative examples shown in Figs. 4 and 8) will inevitably affect any further nanostructure growth.

Even though both lower- $U_{s}$ (Sec. IV A) and higher- $U_{s}$ (Sec. IV B) conditions create nonuniformities in the ion current profiles, there are remarkable differences between the two cases. In particular, comparing the distributions of the relative ion current density along the nanotip lateral surfaces (Figs. 5 and 9), one can clearly see that in the wide sheath case the distribution is strongly nonuniform, in a remarkable contrast to the small sheath case featuring fairly uniform ion flux deposition. Physically, the nanotip growth critically depends on the location of the maximum of the ion current on the lateral nanotip surface. The displacement of the current maximum at higher plasma densities suggests that the actual shape and growth kinetics of the nanostructures are strongly affected by the value of $n_{p}$. In dense plasmas, one could expect a preferential growth of the nanotip base and hence, the formation of wider and shorter nanoassemblies. On the other hand, the low-density plasma conditions favor the development of the nanotips with a larger aspect ratio. It is notable that the narrow sheath case shows a fairly uniform and regular distribution of the ion current over the entire simulation area. It should be expected that the nanotips grown under such conditions would not change their shape significantly in the growth process. This conclusion is consistent with the experimental results on the CNS growth shown in Fig. 12 and earlier studies of the CNS growth kinetics ${ }^{34}$

This clear difference in the ion current distribution can be explained by invoking the following arguments. Under the wide sheath conditions (Sec. IV B), when the current is distributed nonuniformly along the lateral nanotip surface, the ion approaches the sharp end of the "host" nanotip (where the electric field is strongly nonuniform) with a relatively high velocity. Thus, $J_{s}$ is less affected by the weak electric fields created by the neighboring nanotips, and the

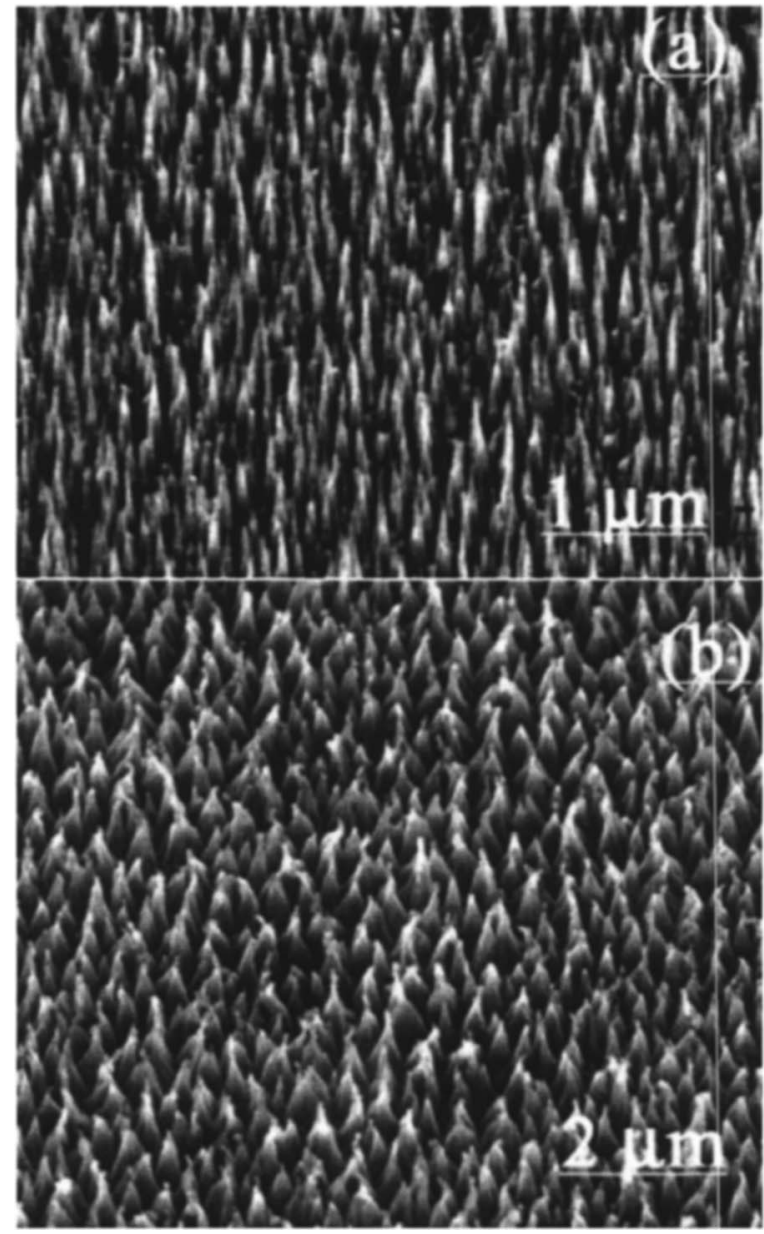

FIG. 12. Representative high-resolution scanning electron micrographs of ordered patterns of carbon nanostructures for electron microemitter applications in flat display technology (courtesy of Z. L. Tsakadze, J. D. Long, K. Ostrikov, and $\mathrm{S}$. $\mathrm{Xu}$ ). Vertical and horizontal dimensions of singlecrystalline carbon nanotips [top figure, $h=250-350 \mathrm{~nm}, R=30-60 \mathrm{~nm}$ (at $z=h / 2)$ ] and carbon nanopyramidal structures [bottom figure, $h$ $=350-500 \mathrm{~nm}, R=70-120 \mathrm{~nm}($ at $z=0)]$ appear to be remarkably uniform over large deposition areas $\left(\sim 10 \mathrm{~cm}^{2}\right)$, which is consistent with the results of Sec. III. Recent reports on electron field-emission currents (see Ref. 34) suggest an outstanding potential of the ordered single-crystalline nanotip and nanopyramidal structures for the development of microemitter display panels.

ion motion is mainly controlled by the electric field of the host nanotip. As a result, the ions precipitate locally on the surfaces of their host nanostructures, which results in somewhat diffuse but nevertheless pronounced maxima in the ion current distribution.

When the plasma sheath is narrow (Sec. IV A), the ion approaches the nanotip pattern with a smaller velocity. In this case, weak electric fields created by the neighboring nanotips distort the trajectory appreciably, which dithers the ion flux and causes fairly uniform ion current distributions. The dependence of the relative ion current density on the nanotip radius (Figs. 6 and 10) suggest the possibility to control the distribution of nanotip radii [with the initial nanotip radii distribution function (NRDF) shown in Fig. 3] in the process of nanofabrication of the ordered microemitter pattern (Fig. 2). Evidently, preferential ion deposition on small nanotips can enhance their growth and, hence, contribute to the 
smoothening of the NRDF. This effect is strongly pronounced for both low- $U_{s}$ and high- $U_{s}$ conditions.

Therefore, PECVD of CNSs in higher-density plasmas is advantageous for the assembly of microemitter arrays with highly uniform size distribution (e.g., NRDF) of individual nanostructures. Remarkably, in the experiments on the CNS synthesis in low-temperature rf plasmas of $\mathrm{Ar}+\mathrm{H}_{2}+\mathrm{CH}_{4}$ mixtures the combined ion number densities exceeded $\sim 10^{18} \mathrm{~m}^{-3} \cdot{ }^{33-35}$ As has been discussed above, the most likely causes of the NRDF reshaping during the growth process is the more efficient focusing of the electric field and thus ion attraction by smaller nanotips. At higher plasma densities, this effect becomes stronger due to the smaller sheath thickness. One can thus expect a higher degree of the NRDF equalization under denser plasma conditions.

The dependence of the mean relative ion current density onto the nanotips on the plasma density [Figs. 7(a) and 11(a)] reveals the possibility to control the nanotip density on the substrate. Indeed, the concentration of the adsorbed atoms on the substrate surface determines the rate of the island nucleation. ${ }^{48}$ Let us consider what it means in terms of CNS growth kinetics and assume that during the first growth stage a certain nanotip pattern (with the areas covered and free of nanotips) has been formed. When the ion current density onto the nanotips is high, one can expect that the nucleation rate of adatoms on the nanotip-free surface areas will be somewhat reduced promoting preferential growth of the already existing nanostructures. Furthermore, the surface areas between the nanotips should feature a smaller amount of irregular growth islands and thus a smoother surface morphology.

On the other hand, when the relative ion current density to the nanotips is close to $J_{s}^{m}$, one can expect a higher density of the adsorbed atoms in the nanotip-free areas, and hence, more efficient nucleation and growth island development. The resulting pattern will thus feature quite different surface morphologies, with somewhat shorter nanotip structures and a number of unwelcome buildups in the internanotip areas. If the areas uncovered by the nanotips contain catalyst residues (this can happen when the $\mathrm{Ni} / \mathrm{Fe} / \mathrm{Co}$ catalyst layer fragmentation during the surface activation stage is incomplete ${ }^{34}$ ), the CNSs can also start growing in the internanotip surface areas and eventually interfere with the ordered nanotip pattern. Furthermore, this can result in an uncontrollable growth of the nanotips with a large difference in height over the pattern.

The dependence of the mean relative ion current density onto the nanotips on the electron temperature [Figs. 7(b) and 11(b)] appears to be very different for low- $U_{s}$ and high- $U_{s}$ conditions. When $U_{s}=20 \mathrm{~V}$, the relative current density decreases with the electron temperature, whereas the inverse dependence is observed when the cross-sheath potential drop is $50 \mathrm{~V}$. This phenomenon can be explained by noting that the dependence of the sheath thickness on $T_{e}$ is quite different under the narrow- and wide-sheath conditions. Indeed, comparing Eqs. (1) and (2), one can notice that $\lambda_{s} \propto T_{e}^{-1 / 4}$ in the high- $U_{s}$ case and $\lambda_{s} \propto T_{e}^{1 / 2}$ otherwise. Thus, different scalings of the sheath thickness with the electron temperature result in a quite different behavior of the relative ion current density onto the nanotips. The electron temperature can thus be regarded as another useful control knob of the growth kinetics of the ordered carbon nanotip structures. From Figs. 7(b) and 11(b), one can see that the strongest dependence of the relative current density to the nanotips on $T_{e}$ is in the range below $2-3 \mathrm{eV}$, which is a typical electron temperature in the CNS synthesis experiments of our interest here. ${ }^{33-35}$

The effect of nonuniform ion fluxes on the adatom surface diffusion and incorporation into the nanotip structures being grown has been sidestepped here. Thus, the growth mechanism of the carbon nanotips cannot be explained merely based on the results presented here. Elaboration of the details of the nanotip growth kinetics will be a subject of our future research efforts. Nonetheless, the results of this work enable us to presume that the nanosized grain growth ${ }^{49}$ and eventually the CNS pattern are strongly affected by the nonuniform ion fluxes, which can lead to a notable rearrangement of the growth patterns and the resulting nanoscale surface morphology. ${ }^{50}$

To conclude this section, we emphasize that despite a close relevance of this work to the specific plasma-aided nanofabrication process employing hydrocarbon-based reactive plasmas and specific building units (being $\mathrm{CH}_{3}^{+}$here), the results of this work are quite generic and the main conclusions can be applied to a broader range of ordered nanotip structures. Our model can straightforwardly be extended into different plasma chemistries, complex plasmas, ${ }^{51}$ deposition of multiple cationic species, denser ion fluxes and account for ion collisions within the plasma sheath, and broader ranges of substrate bias and other process control parameters to fit the demands of various reactive plasma-assisted nanofabrication processes.

\section{CONCLUSION}

Three-dimensional topography of the microscopic ion current onto the ordered array of vertically aligned carbon nanotips has been studied by the Monte Carlo simulation in two different cases of lower and higher electrostatic potential drops across the plasma sheath. When the substrate is unbiased or floating (which corresponds to the low- $U_{s}$ case), the nanotip array focuses the ion flux more efficiently than in the higher- $U_{s}$ case (dc-biased substrate). In both cases, the relative current density onto the nanotips depends strongly on the plasma density and electron temperature. The nanotip aspect ratio appears to be another important factor, since the ion flux focusing by smaller-radius nanotips appears to be more efficient. Likewise, the distribution of the ion current along the nanotip lateral surface is strongly nonuniform and can be controlled by the plasma density. Our results suggest that the plasma parameters and substrate bias are the important factors that enable one to efficiently manipulate the microscopic ion fluxes onto the substrate and nanotip surfaces, eventually leading to the possibility of the efficient carbon nanotip growth control. Finally, the main challenge for future work is to translate the knowledge obtained into industrially viable process control strategies for nanomanufacturing of uniform and vertically aligned patterns of single-crystalline carbon microemitters. 


\section{ACKNOWLEDGMENTS}

This work was partially supported by the Australian Research Council, the University of Sydney, the Agency for Science, Technology and Research (Singapore), and the International Research Network for Deterministic PlasmaAided Nanofabrication. Two of the authors (K.O.) and (S.X.) thank J. D. Long and Z. L. Tsakadze for collaboration on the plasma-assisted growth of carbon nanotip and nanopyramid microemitters and unpublished scanning electron microscopy (SEM) micrographs of the relevant nanostructures.

${ }^{1}$ Market research by iSuppli/Stanford Resources, El Segundo, CA; http:// www.isuppli.com

${ }^{2}$ M. S. Dresselhaus, G. Dresselhaus, and P. C. Eklund, Science of Fullerenes and Carbon Nanotubes (Academic, San Diego, CA, 1996).

${ }^{3}$ W. P. Kang, T. Fisher, and J. L. Davidson, New Diamond Front. Carbon Technol. 11, 129 (2001).

${ }^{4}$ E. T. Thostenson, Z. Ren, and T. W. Chou, Compos. Sci. Technol. 61, 1899 (2001).

${ }^{5}$ K. B. K. Teo et al., IEE Proc.-G: Circuits, Devices Syst. 151, 443 (2004).

${ }^{6}$ L. Nilsson, O. Groening, O. Kuettel, P. Groeniing, and L. Schlapbach, J. Vac. Sci. Technol. A 20, 326 (2002).

${ }^{7}$ M. Mauger, V. T. Bihn, A. Levesque, and D. Guillot, Appl. Phys. Lett. 85, 305 (2004).

${ }^{8}$ K. N. Tu, J. W. Mayer, and L. C. Feldman, Electronic Thin Film Science - for Electrical Engineers and Materials Scientists (Macmillan, New York, 1992).

${ }^{9}$ D. G. Schlom, J. H. Haeni, J. Lettieri, C. D. Theis, W. Tian, J. C. Jiang, and X. Q. Pan, Mater. Sci. Eng., B 87, 282 (2001).

${ }^{10}$ Structure-Property Relationships of Oxide Surfaces and Interfaces, edited by C. B. Carter, X. Q. Pan, K. Sickafus, H. L. Tuller, and T. Wood (Materials Research Society, Warrendale, 2001), Vol. 654.

${ }^{11}$ B. T. Liu et al., Appl. Phys. Lett. 80, 4801 (2002).

${ }^{12}$ S. Somiya, H. Toyoda, Y. Hotta, and H. Sugai, Jpn. J. Appl. Phys., Part 1 43, 7696 (2004).

${ }^{13}$ H. Toyoda, H. Morishima, R. Fukute, Y. Hori, I. Murakami, and H. Sugai, J. Appl. Phys. 95, 5172 (2004).

${ }^{14}$ W. B. Choi et al., Appl. Phys. Lett. 75, 3129 (1999).

${ }^{15}$ C. Bower, W. Zhu, S. Jin, and O. Zhou, Appl. Phys. Lett. 77, 830 (2000).

${ }^{16}$ M. Chhowalla et al., J. Appl. Phys. 90, 5308 (2001).

${ }^{17}$ C. L. Tsai, C. F. Chen, and L. K. Wu, Appl. Phys. Lett. 81, 721 (2002).

${ }^{18}$ S. B. Lee, A. S. Teh, K. B. K. Teo, M. Chhowalla, D. G. Hasko, W. I. Milne, G. A. J. Amaratunga, and H. Ahmed, Nanotechnology 14, 192 (2003).

${ }^{19}$ M. Meyyappan, L. Delzeit, A. Cassel, and D. Hash, Plasma Sources Sci. Technol. 12, 205 (2003).

${ }^{20}$ S. Hofmann, C. Dukati, J. Robertson, and B. Kleinsorge, Appl. Phys. Lett. 83, 135 (2003).

${ }^{21}$ Y. Shiratori, H. Hiraoka, Y. Takeuchi, S. Itoh, and M. Yamamoto, Appl. Phys. Lett. 82, 2485 (2003).

${ }^{22}$ C. Ducati, I. Alexandrou, M. Chhowalla, J. Robertson, and G. A. J. Amaratunga, J. Appl. Phys. 95, 6387 (2004)
${ }^{23}$ M. S. Bell, R. G. Lacerda, K. B. K. Teo, N. L. Rupesinghe, G. A. J. Amaratunga, W. I. Milne, and M. Chhowalla, Appl. Phys. Lett. 85, 1137 (2004).

${ }^{24}$ K. Ostrikov, Z. Tsakadze, P. P. Rutkevych, J. D. Long, S. Xu, and I. Denysenko, Contrib. Plasma Phys. 45, 514 (2005); K. Ostrikov, I. Denysenko, M. Y. Yu, and S. Xu, Phys. Scr. 72, 277 (2005).

${ }^{25}$ K. N. Tu, J. Appl. Phys. 94, 5451 (2003).

${ }^{26}$ K. Ostrikov, Rev. Mod. Phys. 77, 489 (2005).

${ }^{27}$ Nanotechnology Research Directions: Vision for Nanotechnology Research and Development in the Next Decade edited by M. C. Roco, S. Williams, and P. Alivisatos (Kluwer Academic, Amsterdam, 1999); See also: US National Nanotechnology Initiative, http://www.nano.gov.

${ }^{28}$ C. P. Poole, Jr. and F. J. Owens, Introduction to Nanotechnology (Wiley, New York, 2003).

${ }^{29}$ V. Shchukin, N. N. Ledentsov, and D. Bimberg, Epitaxy of Nanostructures (Springer, Berlin, 2003).

${ }^{30} \mathrm{H}$. L. Chua and S. Xu, Second International Conference on Nanostructures and Nanotechnology, Singapore, 25-26 November 2004 (unpublished), Contrib. Paper T18.

${ }^{31}$ A. Fridman and L. A. Kennedy, Plasma Physics and Engineering (Taylor \& Francis, New York, 2004).

${ }^{32}$ M. A. Lieberman and A. J. Lichtenberg, Principles of Plasma Discharges and Materials Processing (Wiley, New York, 1994).

${ }^{33}$ I. B. Denysenko, S. Xu, P. P. Rutkevych, J. D. Long, N. A. Azarenkov, and K. Ostrikov, J. Appl. Phys. 95, 2713 (2004).

${ }^{34}$ Z. L. Tsakadze, K. Ostrikov, J. D. Long, and S. Xu, Diamond Relat. Mater. 13, 1923 (2004).

${ }^{35}$ Z. L. Tsakadze, K. Ostrikov, and S. Xu, Surf. Coat. Technol. 191/1, 49 (2005).

${ }^{36}$ I. Levchenko, M. Romanov, and M. Keidar, J. Appl. Phys. 94, 1408 (2003).

${ }^{37}$ D. Hash and M. Meyyappan, J. Appl. Phys. 93, 750 (2003).

${ }^{38}$ D. Hash, D. Bose, T. R. Govindan, and M. Meyyappan, J. Appl. Phys. 93, 6284 (2003).

${ }^{39}$ F. J. Gordillo-Vazques and J. M. Albella, Plasma Sources Sci. Technol. 13, 50 (2004).

${ }^{40}$ E. I. Waldorff, A. M. Waas, P. P. Friedmann, and M. Keidar, J. Appl. Phys. 95, 2749 (2004).

${ }^{41}$ A. N. Obraztsov, I. Pavlovsky, A. P. Volkov, E. D. Obraztsova, A. L. Chuvilin, and V. L. Kuznetsov, J. Vac. Sci. Technol. B 18, 1059 (2000).

${ }^{42}$ E. Abdel-Fattah and H. Sugai, Appl. Phys. Lett. 83, 1533 (2003).

${ }^{43}$ H. Sugai, I. Ghanashev, and M. Nagatsu, Plasma Sources Sci. Technol. 7, 192 (1998).

${ }^{44}$ S. Xu, K. N. Ostrikov, Y. Li, E. L. Tsakadze, and I. R. Jones, Phys. Plasmas 8, 2549 (2001).

${ }^{45}$ J. D. Jackson, Classical Electrodynamics (Wiley, New York, 1967).

${ }^{46}$ I. Levchenko, M. Korobov, M. Romanov, and M. Keidar, J. Phys. D 37, 1619 (2004).

${ }^{47}$ A. Klein and A. Godunov, Introductory Computational Physics (Cambridge University Press, Cambridge, UK, 2005).

${ }^{48}$ Q. Y. Zhang and P. Chu, Surf. Coat. Technol. 158, 247 (2002).

${ }^{49}$ K. N. Tu, A. M. Gusak, and I. Sobchenko, Phys. Rev. B 67, 245408 (2003).

${ }^{50}$ L. Levchenko and O. Baranov, Vacuum 72, 205 (2004).

${ }^{51}$ S. V. Vladimirov and K. Ostrikov, Phys. Rep. 393, 175 (2004). 